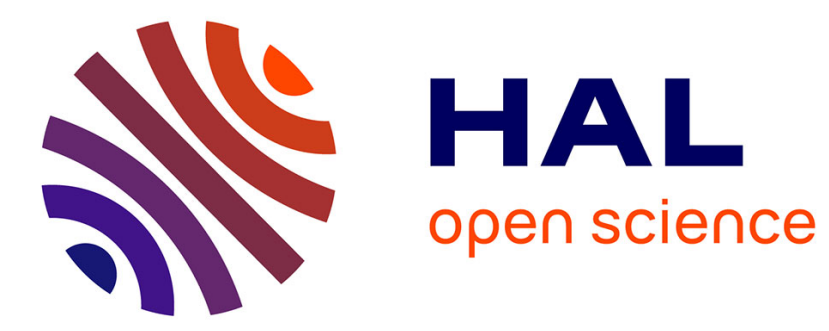

\title{
Structure of vacancy induced spin polarons in solid $3 \mathrm{He}$
} G. Montambaux, P. Lederer, M. Héritier

\section{To cite this version:}

G. Montambaux, P. Lederer, M. Héritier. Structure of vacancy induced spin polarons in solid 3He. Journal de Physique Lettres, 1979, 40 (18), pp.499-503. 10.1051/jphyslet:019790040018049900 . jpa00231674

\section{HAL Id: jpa-00231674 https://hal.science/jpa-00231674}

Submitted on 1 Jan 1979

HAL is a multi-disciplinary open access archive for the deposit and dissemination of scientific research documents, whether they are published or not. The documents may come from teaching and research institutions in France or abroad, or from public or private research centers.
L'archive ouverte pluridisciplinaire HAL, est destinée au dépôt et à la diffusion de documents scientifiques de niveau recherche, publiés ou non, émanant des établissements d'enseignement et de recherche français ou étrangers, des laboratoires publics ou privés. 


\title{
Structure of vacancy induced spin polarons in solid ${ }^{3} \mathrm{He}$
}

\author{
G. Montambaux, P. Lederer and M. Héritier \\ Laboratoire de Physique des Solides $\left({ }^{*}\right)$, Université Paris-Sud, Centre d'Orsay, 91405 Orsay, France
}

(Reçu le 27 février 1979, révisé le 18 juillet 1979, accepté le 24 juillet 1979)

\begin{abstract}
Résumé. - On étudie l'existence de polarons ferromagnétiques induits par des lacunes, dans les phases cubiques centrées et hexagonales bidimensionnelles de ${ }^{3} \mathrm{He}$. On discute l'hypothèse de parfaite localisation et la limite des polarons de petite taille. Ceux-ci ne paraissent pas pouvoir expliquer le défaut d'entropie observé autour de $1 \mathrm{~K}$ dans ${ }^{3} \mathrm{He}$ cubique centré. On discute ensuite la possibilité de la condensation des polarons en une goutte ferromagnétique macroscopique.
\end{abstract}

Abstract. - We study the existence of ferromagnetic polarons induced by vacancies, in b.c.c. and $2 \mathrm{D}$ hexagonal
${ }^{3} \mathrm{He}$. We discuss the assumption of perfect vacancy localization within the polaron and the small size polaron
limit. These models do not seem to account for some negative contribution to the entropy observed around $1 \mathrm{~K}$
in b.c.c. ${ }^{3} \mathrm{He}$. We then discuss the possibility of polaron condensation in a macroscopic ferromagnetic drop.

1. Introduction. - Giant spin polarons are thought to form around vacancies in fermion quantum crystals such as b.c.c. ${ }^{3} \mathrm{He}$ or $2 \mathrm{D}$ alternate phases $[1,2]$. The vacancy lowers its kinetic energy by propagating in a region of lined-up spins, the volume of which is limited by the entropy and exchange energy loss.

It may be that susceptibility [2] and specific heat anomalies [3] which have been observed in the $\mathrm{mK}$ region [4] in b.c.c. ${ }^{3} \mathrm{He}$ are experimental manifestations of these polarons, although other theoretical explanations are available in the literature [4]. Irrespective of the actual validity of theoretical treatments of the observed anomalies, the existence of vacancy magnetic polarons under convenient physical conditions can hardly be questioned, as it relies on a few essential features of fermion quantum crystals.

Theorical treatments until now $[1,2]$ have been based on a very simple model which treats the lattice as a continuum, which is strictly correct only for infinitely large polarons. Up to now, conditions for the existence of polarons as a function of temperature and strength of exchange forces have been discussed only briefly [2]. The implication of the alternate or non-alternate character of the lattice have been stressed recently [5].

It appears to be a convenient time, in view of the progress of low temperature techniques and of experiments on ${ }^{3} \mathrm{He}$ in the relevant range of tempe-

(*) Laboratoire associé au C.N.R.S. rature $[4,6]$ to refine existing models, test their validity, and make a more accurate assessment for polaron thermodynamics [7]. This paper deals with :

i) the effects linked with the polaron boundary,

ii) the small size polaron limit which would seem appropriate to higher temperature,

iii) the possibility of polaron condensation in a macroscopic ferromagnetic drop [7].

The first topic has either been disregarded in previous work [1] or discussed very briefly [2]. The second topic has not been discussed previously. We discuss the third aspect either at fixed number of particles or at fixed chemical potential, and the possibility of an experimental observation.

2. Review. - We study a crystal with nearly one fermion per site, with a repulsive interaction $U$ which we suppose is infinite. We consider the case of a finite temperature $T$. We neglect orbital degeneracy. The relevant Hamiltonian is the Hubbard Hamiltonian which allows an approximate description of exchange forces. Elastic properties of the lattice are not well described. But essential properties due to the antisymmetrization of the whole wave function of the lattice of fermions with a hard core repulsive interaction are well accounted for, when hole propagation through the crystal is involved.

In the infinite repulsive interaction limit, configurations with two fermions on the same site are for- 
bidden. A hole propagates through the lattice by exchanging its place with a nearest neighbouring fermion. Its motion depends on the magnetic configuration. A theorem due to Nagaoka [8] shows that, in this case, the ground state is the fully ferromagnetic state. The hole moves in an energy band stretching from $-z t$ to $+z t$ where $z$ is the number of nearest neighbours and $t$ is the overlap integral between nearest neighbours.

An extension of this theorem, due to Brinkman and Rice [9], shows that for a random configuration the vacancy band is narrowed. It extends from $-\omega_{0} z t$ to $\omega_{0} z t$ where

$$
\omega_{0}=2 \frac{\sqrt{z-1}}{z}
$$

in the self-retracing-paths approximation [9]. It is a consequence of the partial localization of the vacancy because of the suppression of paths for its propagation.

Several authors $[1,2]$ suppose that at finite temperature, the vacancy decreases its kinetic energy by localizing itself in a ferromagnetic polaron, the size of which is limited by entropy and exchange (we suppose here that exchange is negligible if $U \gg t$ ). In order to reduce the many body problem to a single particle problem, they proposed the model of a sphere of radius $R$, within which the spin polarization $\left\langle S_{z}\right\rangle$ is supposed to be uniform. $\left\langle S_{z}\right\rangle$ varies abruptly from $\frac{1}{2}$ to 0 through the polaron boundary.

In fact the vacancy is not entirely localized in the ferromagnetic volume since the potential energy is not infinite outside the polaron. There still exist paths in the spin disordered region for which the vacancy motion does not perturb the spin configuration. Formally the problem is somewhat different from the localization of an electron in a bubble in liquid ${ }^{4} \mathrm{He}[10,11]$, a physical situation which offers an example of a nearly perfectly localized particle. In this case the depth of the potential well in which the electron moves is quasi infinite compared with the ground state energy of the particle.

Therefore in our case, we have to take into account the exponential decay of the vacancy wave function outside the polaron [2] and we shall show the importance of the boundary effects on the previously obtained results. Furthermore we shall extend these results in the limit of small size polarons $(R \simeq a)$ where the discrete nature of the lattice cannot be ignored. In that case, the kinetic energy can be evaluated by the moment method [9].

3. Localization energy. - In one dimension, all paths are self retracing and the motion of the vacancy does not perturb the surrounding spins. Thus there does not exist spin polaron in one dimension.

In two or three dimensions, the existence of a ferromagnetic polaron is possible in an alternate lattice [5, 7]. In this case, the lowest kinetic energy of the hole is assumed to be the lowest bound state in the potential

$$
\begin{array}{lll}
V(r)=-z t & \text { when } & r<R \\
V(r)=-\omega_{0} z t & \text { when } & r>R .
\end{array}
$$

This effective potential expresses, in an approximate way, the fact that the kinetic energy of the vacancy depends on the spin configuration. The ground state corresponds to the $l=0$ orbital number. We have to solve the Schrödinger equation for a particle of mass $m$ moving in $V(r)$.

In a totally ferromagnetic medium, the problem would be a single particle problem with $k$ a good quantum number. The effective mass depends on the transfer integral $t$ through the dispersion relation obtained by the tight-binding method. An expression of these relations is possible near the zone boundary :

$$
\begin{array}{ll}
\hbar^{2} / 2 m=\frac{3}{4} a^{2} t & \text { for the } 2 \mathrm{D} \text { hexagonal lattice } \\
\hbar^{2} / 2 m=\frac{4}{3} a^{2} t & \text { for the b.c.c. lattice }
\end{array}
$$

where $a$ is the distance between nearest neighbours. In fact the nuclear spin system is perturbed when the vacancy tunnels from site to site. For that reason, the study of the vacancy cannot be reduced to a single particle problem and $k$ is not a good quantum number. Therefore considering the evolution of a particle in an effective potential is a serious approximation, in particular when the polaron size is comparable with the lattice spacing.

The kinetic energy of the particle is written

$$
E=\frac{\hbar^{2}}{2 m R^{2}} y^{2}-z t
$$

In the perfect localization limit, $y$ equals $\pi$ in three dimensions and 2.405 (first zero of $J_{0}$, the first Bessel function of the first kind) in two dimensions. Figure 1 shows the variation of $E$ versus $R$ by solving Schrödinger equation for the potential (1) in $3 \mathrm{D}$. In two dimensions, there always exists a bound state whatever the polaron radius $R$ may be. The vacancy energy goes to $-\omega_{0} z t$ when $R$ goes to zero. In three dimensions the bound state disappears if

$$
V=\frac{\hbar^{2}}{2 m R^{2}}\left(\frac{\pi}{2}\right)^{2} \text { i.e. } \frac{R_{0}}{a}=\frac{\pi}{\sqrt{3}} \sqrt{\frac{t}{V}} \simeq 1.3
$$

for the b.c.c. lattice. For $R<R_{0}$, the kinetic energy is equal to the energy of the upper edge of the well, i.e., the energy in the random medium, $-\omega_{0} z t$. This difference is probably unphysical and stems from the continuum approximation.

One can see that the exact solution of the continuum problem differs appreciably from the simplest one in which a perfect localization is assumed [1]. Edge effects lower the kinetic energy, particularly for small polaron radii. For $R \approx 2$, a case of physical 
interest $[2,3]$, the exact energy is smaller by a factor of 2 .

However, for these small size polarons, the discreteness of the lattice cannot be ignored. Therefore, we have used a moment method. We have computed the first few moments of the vacancy density of states in two dimensions (up to the 10th moment) and in three dimensions (up to the 8th moment) for polarons of different sizes. The difficulty of the nethod increases tremendously with the order of the moment, because we need to know, for each path, the probability that the initial spin configuration is restored at the end of the path $[9,12]$. By extrapolation of the first moments, we have obtained the lower band edge energy of the vacancy. Given the small number of moments we have computed, the determination implies large error bars (see Fig. 1). We find that the continuum approximation is, by chance, surprisingly good at very small radii. However, for $R \sim 2$, the kinetic energy in the discrete lattice is smaller than in the continuum.

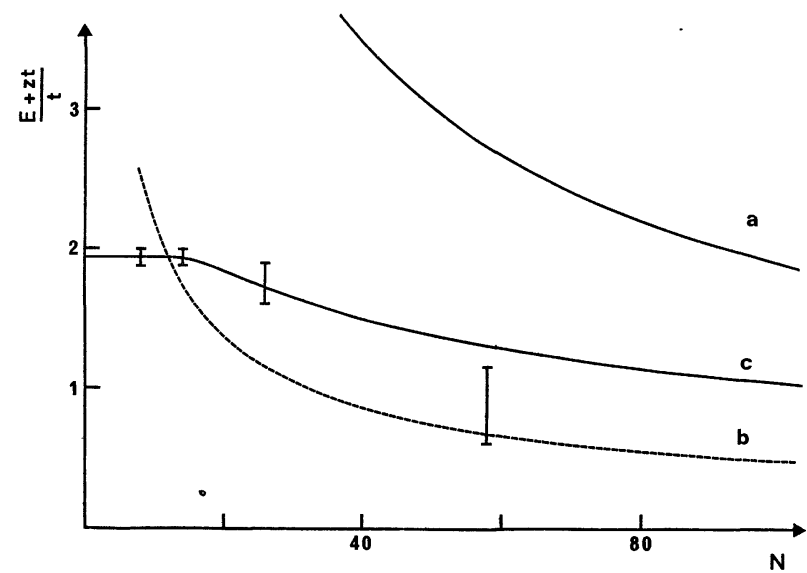

Fig. 1. - Localization energy of a vacancy in a polaron in the b.c.c. lattice versus the number $N$ of spins in the polaron. One particle continuum approximation : $a$ ) in a perfect localization model, $\left.E=\pi^{2} \times \hbar^{2} / 2 m R^{2}, b\right) E=\pi^{2} / 4 \times \hbar^{2} 2 m R^{2}, c$ ) exact calculation. The bound state disappears if $N \simeq 12$. The bars give an estimation of polaron energy calculated by extrapolation of the first moments of the density of states.

4. Existence of the polaron state. - Mott [13] estimates that the vacancy band shows strong peaks due to the heavy spin polaron. An exact calculation of the free energy requires the knowledge of the form of the density of states for one particle. In a simple calculation, various authors $[1,2,5,7,13]$ assumed that vacancies are all in the ground state. We also make this assumption which we shall discuss below.

Taking these approximations and in a model of independent vacancies, the free energy is

$F=-z t+\frac{\hbar^{2} y^{2}}{2 m R^{2}}+N k_{\mathrm{B}} T \ln 2-k_{\mathrm{B}} T \ln (N+1)$.
For each structure, the depth of the potential well $V$ is given, $y$ and $E$ depend only on the radius $R$. The first term describes the lower energy state for the vacancy in the ferromagnetic medium. In the continuum approximation, the second one is the energy to localize partially the vacancy in the sphere (finite potential well). The third one is the entropy increase with respect to a totally random region. The spherical model considered here will be quantitatively valid if $R$ is large enough. In this case the number $N$ of spins in the polaron is in the $2 \mathrm{D}$ hexagonal lattice :

$$
N=\frac{4 \pi}{3 \sqrt{3}} \frac{R^{2}}{a^{2}}
$$

and in the b.c.c. lattice :

$$
N=\pi \sqrt{3} \frac{R^{3}}{a^{3}}
$$

where $a$ is the distance between nearest neighbours. If $N$ is large enough so that the continuum approach is valid, we neglect the term $\ln (N+1)$.

The free energy is a function $F(R, T)$ of the radius $R$ and of the temperature $T$. It always has a local minimum at $R=0$ which does not appear in a perfect localization model. It generally exhibits another minimum for $R=R_{\mathrm{m}}(T)$ which describes the polaron state. This second minimum is below the first one $\left(-\omega_{0} z t\right)$ above a critical temperature $T_{\mathrm{c}}$. Then if $T<T_{\mathrm{c}}$ the stable state is a polaron of radius $R_{\mathrm{m}}(T)$. The parameters $T_{\mathrm{c}}$ and $R_{\mathrm{m}}(T)$ are given by the variational treatment. The polaron exists for temperature $T$ as

$$
F_{\mathrm{m}}(T)=F\left(R_{\mathrm{m}}(T), T\right)<F(0, T) .
$$

- $2 \mathrm{D}$ hexagonal lattice : the self retracing paths approximation is rather valid since the relative error in the sixth moment is $4 \times 10^{-3}$. Then with a good approximation, we get

$$
V=(z-2 \sqrt{z-1}) t=(3-2 \sqrt{2}) t=0.17 t .
$$

The validity of this approximation is due to the small number of nearest neighbours which implies large loops. The exact variational calculation has to take into account the variation of $y$ versus the radius $R$; $y$ is always smaller than 2.405 found by Feigel'man [2]. The minimization of the free energy with respect to $R$ gives the variation of $F_{\mathrm{m}}(T)$ versus $T$. The critical temperature below which the polaron becomes thermodynamically stable is

$$
T_{\mathrm{c}} \approx 2.5 \times 10^{-3} t
$$

At this temperature the radius of the sphere is about

$$
R \approx 3.5 a \text {. }
$$

The polaron has roughly 30 atoms. The spherical 
approximation is good enough for this temperature region.

- b.c.c. lattice : the self-retracing-paths approximation is less good. It leads to

$$
V=(8-2 \sqrt{7}) t=2.71 t
$$

A moment calculation gives more exactly the random bandwidth and leads to

$$
V \simeq 8(1-0.76) t=1.94 t
$$

In a better treatment we have to use this result. As in two dimensions, the critical conditions are obtained with the condition (4)

$$
\begin{aligned}
T_{\mathrm{c}} & \simeq 1.3 \times 10^{-2} t \\
R & \simeq 2.1 \mathrm{a} \\
N & \simeq 50 \text { spins } .
\end{aligned}
$$

The true value of $T_{\mathrm{c}}$ is expected to be slightly larger. We find by the moment method that a polaron of 58 spins is stable for $T<3 \times 10^{-2} t$.

Another difficulty is due to the fact that the ground state is not the only occupied state. So we have to know the form of the density of states in the two configurations. In the random one, Brinkman and Rice [9] found a band of the form

$$
\rho(\omega)=C\left(\left(\omega_{0} z t\right)^{2}-\omega^{2}\right)^{v} .
$$

If we consider that the band structure is not significantly altered by passing from the random configuration to the polaron configuration, the temperature $T_{\mathrm{c}}$ is nearly unchanged. But if all states condense in a polaron peak, $T_{\mathrm{c}}$ increases by one order of magnitude. The exact determination of $T_{\mathrm{c}}$ requires a detailed knowledge of the vacancy band.

5. Remarks on the condensation in a ferromagnetic macroscopic drop. - Feigelman [7] studies submonolayers films of ${ }^{3} \mathrm{He}$ adsorbed on a graphite surface. $\mathrm{He}$ assumes that in a lattice commensurable with the substrate, the number of vacancies is fixed. $\mathrm{He}$ predicts that the condensation of the polarons in a ferromagnetic macroscopic drop decreases the free energy of the vacancies by a multiplicative term of order 1: $F_{\mathrm{m}}<F_{\mathrm{m}}$. In his treatment he considers that $y=2.405$ is constant. This has to be corrected by boundary effects. $y$ is in fact a decreasing function $y(T)$ of the temperature always lower than 2.405 . A rigorous calculation gives

$$
F_{\mathrm{m}}=\tilde{F}_{\mathrm{m}} \times y .
$$

If $y\left(T_{0}\right)=1$, the polaron state becomes stable. In fact this happens at a temperature $T_{0}$ where neither polarons nor the drop exist.

Such a condensation is impossible with thermal vacancies. At fixed chemical potential, the vacancies induce either uniform ferromagnetism or ferromagnetic polarons. Even in a commensurate lattice, in thermodynamic equilibrium, the fixed quantity is the vacancy chemical potential, i.e. the external pressure, under usual experimental conditions. Then, a macroscopic region without thermal vacancies cannot be stable, either in a $2 \mathrm{D}$ commensurate layer or in b.c.c. ${ }^{3} \mathrm{He}$.

The case of metastable vacancies might be different. Let us assume that vacancies, at a given temperature, become trapped by absence of diffusion, by forming large magnetic polarons $[2,3]$. Then, at lower temperature, there exists a fixed number of metastable vacancies in excess of the equilibrium concentration. With an increasing applied magnetic field, the vacancy wave function overlap increases, and so does the bandwidth of the sort of impurity band they form. At a critical magnetic field, a Mott-Anderson transition should occur in the impurity band, leaving the vacancies free to diffuse. Then, under suitable conditions, the vacancies could condense and form the Feigelman drop, with an abrupt decrease of the magnetization. Therefore, keeping in mind the number of unproved assumptions, we suggest as a conjecture with the desirable wariness, that the first order transition recently observed at high magnetic fields in b.c.c. ${ }^{3} \mathrm{He}[14]$ is in fact a magnetic polaron condensation.

At fixed chemical potential, what remains of the ferromagnetic drop is a tendency to form multiple vacancy bound states [15] and a finite concentration of di-vacancies, tri-vacancies, etc.

6. Discussion. - The qualitative arguments given in the literature $[1,2]$ about the existence of vacancy induced magnetic polarons can hardly be refuted. However, when one comes to a quantitative description of the polaron in order to compare the experimental data with effects predicted by the theory, a much more refined treatment looks to be necessary.

In particular, we have shown that considering the vacancy as completely localized in the ferromagnetic region is a poor approximation. Obviously, all the simple power laws predicted in the crudest model [1] for the various derivatives of the free energy are not valid. Therefore, our results will change the vacancy contribution to the susceptibility, the specific heat, etc. In the crudest picture [1], a vacancy polaron with less than 200 spins would not be stable. Taking into account the tailing of the vacancy wave function in the disordered medium increases in an important way the stability of the polaron. In the continuum approximation, one finds a minimum size of about 50 spins. When considering the discrete nature of the lattice, this figure is still lowered. We can give an upper limit to the formation temperature $T_{\mathrm{c}}$,

$$
T_{\mathrm{c}}<a \times t
$$

where $a$ is a coefficient certainly smaller than 0.5 . 
Given the range of values proposed for $t(50 \mathrm{mK}$ $500 \mathrm{mK})[16,17]$, the possibility of existence of small size polarons around $1 \mathrm{~K}$, not correctly described in the continuum approximation, is ruled out by our moment calculation. In particular, they could not explain a negative vacancy formation entropy in this temperature range, as recently proposed [18]. Instead, we expect a rapid growth of the polaron around $T_{c}$, to a size of 30 to 50 spins. However, the complete discussion of the polaron formation would require a variational study in which the spin pola- rization $\left\langle S_{z}\right\rangle$ is allowed to vary continuously with temperature.

For temperature near $1 \mathrm{mK}$ in b.c.c. ${ }^{3} \mathrm{He}$, we cannot neglect the exchange $J=0.7 \mathrm{mK}$ on the melting curve [19]. To take it into account we transform terms like $T \operatorname{Ln} 2$ as $T \operatorname{Ln} 2+z J / 4$.

In the $2 \mathrm{D}$ hexagonal lattice, the transfer integral $t$ is probably very small, nearly $5 \mathrm{mK}$ [6]. The polarons will start forming at very low temperature $(10-100 \mu \mathrm{K})$. This temperature region is still experimentally out of reach.

\section{References}

[1] Andreev, A. F., J.E.T.P. Lett. 24 (1977) 564.

[2] Héritier, M. and Lederer, P., J. Physique Lett. 38 (1977) L-209.

[3] Héritier, M. and Lederer, P., J. Physique Colloq. 39 (1978) C6-130.

[4] Landesman, A., J. Physique Colloq. 39 (1978) C6-1305.

[5] Héritier, M. and Lederer, P., Phys. Rev. Lett. 42 (1979) 1068.

[6] Sato, K. and Sugarawa, T., J. Physique Colloq. 39 (1978) C6-281.

[7] Feigelman, M. V., J.E.T.P. Lett. 27 (1978) 491.

[8] Nagaoka, Y., Phys. Rev. 147 (1966) 392.

[9] Brinkman, W. F. and Rice, T. M., Phys. Rev. B 2 (1970) 1324.

[10] Ferrell, R. A., Phys. Rev. 108 (1957) 167.
[11] Kuper, C. G., Phys. Rev. 122 (1962) 1007.

[12] Héritier, M., Thesis, Université Paris-Sud (1975) Orsay.

[13] MotT, N. F., Metal Insulator Transitions (Taylor and Francis Ltd), 1974, p. 135.

[14] Schubert, E. A., Bakalyar, D. M. and Adams, E. D., Phys. Rev. Lett. 42 (1979) 101.

[15] Nozières, P., Private communication.

[16] Sullivan, N., Deville, G. and Landesman, A., Phys. Rev. B 11 (1975) 1858.

[17] Hetherington, J. H., J. Low Temp. Physics 32 (1978) 173.

[18] Widom, A., Sokoloff, J. B. and Sacco, J. E., Phys. Rev. B 18 (1978) 3293

[19] Landesman, A., Ann. Phys. 9 (1975) 69. 